\title{
USO DO FUNGO Fusarium oxysporum COMO INDICADOR DE AMETRINA, ATRAVÉS DA MEDIDA DE BIOMASSA, PELA QUANTIFICAÇÃO DO ERGOSTEROL ${ }^{1}$
}

\begin{abstract}
RESUMO - Neste trabalho avaliou-se o fungo Fusarium oxysporum como potencial bioindicador de contaminação de herbicida da classe das triazinas, por meio do monitoramento do crescimento fúngico. Para tanto, foi avaliado o crescimento micelial em meio de cultura extrato malte e ágar na presença do herbicida ametrina, em diversas concentrações. $\mathrm{O}$ crescimento micelial foi avaliado pela determinação do teor de ergosterol, por CLAE/DAD, em comprimento de onda de
\end{abstract}

\author{
JOSÉ GERALDO GALVÃO ${ }^{2}$ \\ MÁRIO CÉSAR GUERREIRO
JOSEFINA APARECIDA DE SOUZA
JOSE \\ MÁRIO CÉSAR GUERREIRO
JOSEFINA APARECIDA DE SOUZA
JOSE \\ SAMUEL MARTINS DA COSTA COURA ${ }^{5}$
}

$281 \mathrm{~nm}$. A quantificação do ergosterol passa pelas etapas de extração em etanol, saponificação em base forte, fracionamento em hexano:água (4:1) e análise por CLAE. O crescimento do fungo foi retardado pelo aumento da concentração do herbicida, mostrando que o potencial dessa técnica de avaliação de contaminação por ametrina. O ergosterol mostrou ser um bom indicador de biomassa fúngica metabólicamente ativa.

TERMOS PARA INDEXAÇÃO: Ergosterol, ametrina, bioindicador, Fusarium, CLAE.

\section{USING THE FUNGI Fusarium oxysporum AS BIOINDICATOR OF AMETRINE BY MEASURING BIOMASS AND EROGOSTEROL}

\begin{abstract}
In this work the fungi Fusarium oxysporum was evaluated as bioindicator of contamination by a triazine class herbicide, through the screening of the fungal growth. For so, micelial growth measurement was done in culture medium of malt extract and agar in the presence of different concentrations of the herbicide ametrine. The micelial growth measurement was done by the ergosterol
\end{abstract}

content. The ergosterol content was determined by HPLC/DAD, in the wavelength $282 \mathrm{~nm}$, after sample extraction and pre-treatment. The results showed good correlation among fungal biomass and ergosterol content. The fungi growth was reduced by the increase of the ametrine concentration. This shows that using that fungi may be a good alternative for contamination screening.

INDEX TERMS: Ergosterol, ametrine, bioindicator, Fusarium, HPLC.

\section{INTRODUÇÃO}

A necessidade de produtividade na agricultura, com qualidade e menores gastos, levou o homem a utilizar defensivos agrícolas como forma de combate a pragas. O objetivo desses defensivos agrícolas, eliminando pragas e melhorando o rendimento das lavouras, é alcançado, principalmente com o uso de substâncias químicas. Entretanto, o uso intensivo dos diferentes tipos de produtos químicos, principalmente os herbicidas, vêm trazendo consequiências para o homem e o ambiente.

Dessa forma, evidencia-se a necessidade do monitoramento de áreas contaminadas ou de uso contínuo

\footnotetext{
1. Extraído da Dissertação de mestrado do primeiro autor.

2. Mestrando em Agroquímica - Departamento de Agroquímica e Agrobioquímica - UNIVERSIDADE FEDERAL DE LAVRAS/UFLA - Caixa Postal 37 - 37200-000 - Lavras, MG.

3. Químico, DSC, Departamento de Química/UFLA.

4. Pesquisadora do Departamento de Química/UFLA.

5. Acadêmico de Agronomia/UFLA, bolsista de Iniciação Científica/ CNPq.
} 
de defensivos agrícolas, para se obter o conhecimento dos efeitos dessas substâncias e, enfim, entender o impacto sobre o ambiente. Para atender a essa necessidade, deve-se contar com métodos analíticos de sensibilidade e precisão que permitam a avaliação da contaminação de solos e águas por defensivos agrícolas, devendo ter a característica de ser de execução simples, rápida e baixo custo.

Neste trabalho é apresentado um método alternativo de indicação de contaminação de solo por hebicida, utilizando do fungo Fusarium oxysporum como bioindicador, mediante medida de seu crescimento, pela quantificação do ergosterol.

Em estudos preliminares, o F. oxysporum UMC01 (Ellis \& Ellis, 1997) mostrou-se sensível aos herbicidas da classe das triazinas. Segundo Coura et al. (2000), o fungo apresentou alterações na pigmentação e na velocidade de crescimento, quando colocado na presença de diferentes concentrações de herbicida. O seu uso como bioindicador foi considerado promissor para o monitoramento de solos e águas contaminados por herbicidas.

A utilização de bioindicadores, empregando organismos vivos ou parte de suas funções biológicas, é atualmente muito diversificada (Paoletti, 1999). O uso de fungos para monitorar contaminações de solos e águas vem sendo bastante estudado (HölKer et al., 1995; Peintner \& Moser, 1996). A capacidade de biosorção dos fungos aplica-se a metais pesados (Peintner \& Moser, 1996), defensivos agrícolas (Paoletti, 1999), corantes e compostos radioativos (Haselwandter \& Berreck, 1988), entre outros. Sua utilidade advém da produção de metabólitos, que podem sofrer variações em função do meio de cultura, tais como fonte de carbono e de nitrogênio. $\mathrm{O}$ crescimento fúngico pode também sofrer variações, em função do meio e dos metabólitos produzidos (Medentsev \& Akimenko, 1998).

Entre os metabólitos produzidos pelos fungos, destaca-se o ergosterol. Esse esterol é constituinte da membrana plasmática dos fungos, dos quais somente esses produzem tal esterol (Deacon, 1998). O ergosterol é um bom indicador de crescimento fúngico (Anderson et al., 1994), pois pode proporcionar uma boa correlação com a biomassa metabolicamente ativa do fungo. Assim, monitorar a quantidade de ergosterol apresentase como uma boa alternativa para avaliar as alterações de crescimento fúngico. Essas alterações podem, então, ser relacionadas com o efeito causado pela presença de herbicida durante o crescimento do fungo. Dessa maneira, pretende-se monitorar a produção de ergosterol por Cromatografia Líquida de Alta Eficiência (CLAEUV), com a finalidade de viabilizar o uso do $F$. oxysporum como bioindicador da presença de herbicida das classe das triazinas.

\section{Uso de Bioindicadores}

Os bioindicadores são amplamente utilizados para avaliar vários tipos de má administração ambiental, incluindo poluição, uso de produtos agrícolas, descarte incorreto de resíduos, contaminações, etc. (Paoletti, 1999). São vários os trabalhos que evidenciam a utilização de bioindicadores, sendo altamente diversificada, conforme o organismo que se utiliza, seus metabólitos ou suas funções biológicas (Paoletti, 1999).

O mais importante argumento para o uso de organismos vivos como ferramenta para monitorar o ambiente baseia-se no pressuposto de que animais, plantas, microrganismos e suas complexas interações respondem à administração e ao impacto humano por diferentes vias, com alguns organismos respondendo mais rapidamente e definitivamente que outros (Richardson, 1987).

\section{Caracterização do Fungo}

As espécies de Fusarium, organismo-alvo deste trabalho, são freqüentemente encontradas em herbáceas mortas; porém, uma identificação mais acurada necessita da observação de seu crescimento em meio de cultura, onde podem ser observados conídios curvados, septados, estreitos e, freqüentemente, pequenas projeções na base formando os chamados "foot-cells" (pés de célula). Os conídios, fáceis de serem observados, formam massas limosas de cor rosa ou laranja, em forma de almofadas, as chamadas esporodóquios, as quais, sob a forma simples, ou seja, isoladas, são incolores (Ellis \& Ellis, 1997). Os critérios taxonômicos usados, segundo Nelson et al. (1983), na identificação das espécies são: forma basal da célula, tamanho dos macroconídios, presença dos microconídios, tipo de células microconidiogênicas (fiálides) e presença ou não de clamidósporos.

\section{Quantificação de Biomassa Fúngica}

A importância dos fungos para os processos e propriedades dos solos são bem conhecidas; entretanto, a quantificação precisa desses organismos são problemáticas (Parkinson, 1982; Frankland, 1990; Frankland et al., 1990). Métodos acurados e confiáveis para a me- 
dida de biomassa fúngica são essenciais para quantitativamente avaliar seu papel em funções, tais como: decomposição, ciclos de nutrientes, cadeia alimentar e agregação do solo (Christensen, 1989; Newel, 1992).

O monitoramento do desenvolvimento fúngico pode ser feito por métodos biológicos, químicos ou imunológicos. Para a estimativa de biomassa fúngica em solos, várias metodologias são descritas. Esses métodos incluem o uso de componentes bioquímicos específicos das células fúngicas, tais como: quitina, ergosterol, ácidos fosfolipídicos, medidas de atividade metabólicas (inibição seletiva de respiração), contagem direta e contagem por microscopia. Técnicas como coloração de hifas ativas por diacetato de fluoresceína (Soderstron, 1977; Grant \& Harvey, 1982) e ainda o método de detecção de enzimas extracelulares ligadas ao crescimento.

Seitz et al. (1977, 1979) sugerem a análise de ergosterol como uma medida mais apropriada de invasão fúngica de grãos. Ruzicka et al. (2000) vai mais longe, afirmando que a medida da concentração do ergosterol em substratos naturais é, sem dúvida, o mais eficiente método para estimar a biomassa fúngica.

\section{Uso do Ergosterol como Medida de Biomassa Fúngica}

O ergosterol é um bom indicador de crescimento fúngico, pois pode proporcionar uma boa correlação com a biomassa metabolicamente ativa do fungo, uma vez que é um componente da membrana citoplasmática. Vários são os trabalhos que utilizam a quantificação do ergosterol como medida de biomassa fúngica (Seitz et al., 1977, 1979; Ruzicka et al., 2000; Davis \& Lamar, 1992).

O ergosterol, principal esterol fúngico, é um dos componentes da membrana plasmática dos fungos. O principal caminho para a produção do ergosterol e seu percursor é a Acetil-CoA. Todos os esteróis são produzidos pela mesma via básica: com o ácido mevalônico condensando em unidades de isopreno, várias unidades reunindo-se e sofrendo ciclização e diferentes substituições. A importante etapa da via de produção do ergosterol é a remoção de um grupamento metil de um carbono na posição C-14. Essa etapa é catalisada por uma enzima, C-14 demetilase, a qual apresenta um citocromo, como grupo prostético, contendo um átomo de ferro, (Deacon, 1998).

Diferente dos esteróis das plantas superiores, como o sitosterol, o ergosterol tem em sua estrutura um dieno conjugado, portanto, sua absorção específica de luz ultravioleta permite que ele seja identificado e quantitativamente estimado, tanto por espectrofotometria (Martin et al., 1990), quanto por cromatografia líquida de alta eficiência (CLAE), utilizando um detector de ultravioleta com o comprimento de onda de $282 \mathrm{~nm}$ (Goulston \& Mercer, 1969).

\section{Métodos de Extração de Ergosterol}

Os principais métodos de determinação do ergosterol envolvem três estágios: extração da amostra, separação do extrato e análise por cromatografia líquida. Dependendo do substrato, diferentes processos podem ser usados para a extração do ergosterol. Por exemplo, ergosterol pode ser extraído de grãos de cereais invadidos por fungos pela homogeneização em metanol, sendo esse método aplicado em cereais (Seitz et al., 1977), milho e soja (Seitz et al., 1979). Outro exemplo é a extração por saponificação direta em solução alcoólica de hidróxido de potássio, sendo esse método aplicado para extração do ergosterol em raízes, lenhos mortos e solos contaminados com fungos (Davis \& Lamar, 1992).

\section{MATERIAIS E MÉTODOS}

$O$ fungo $F$. oxysporum foi preservado em placas de Petri, contendo $10 \mathrm{~g}$ de solo estéril, à temperatura de $25^{\circ} \mathrm{C}$, obtendo-se assim placas matrizes.

Com o propósito de obter melhores condições na condução dos ensaios biológicos, o fungo $F$. oxysporum foi incubado em meio malte, a $25^{\circ} \mathrm{C}$ durante 7 dias.

Para facilitar a recuperação da massa micelial e visualizar o crescimento e pigmentação do $F$. oxysporum em meio de cultura, uma barreira física de filme de celofane foi colocada entre o fungo e o meio.

\section{Bioensaios em Meio de Cultura na Presença de Herbicida}

Das placas matrizes, uma suspensão de $2 \mathrm{~g}$ de solo e $10 \mathrm{~mL}$ de água (suspensão de esporos) foi inoculada em placas de Petri com meios de cultura [extrato malte $(2 \%)$ e ágar (1,5\%)] e incubada por 7 dias, à temperatura de $25^{\circ} \mathrm{C}$. Após sete dias de incubação, quando a superfície do meio estava totalmente colonizada, foram retirados discos de $5 \mathrm{~mm}$ (inóculo sólido) da região periférica da placa (região de crescimento) e esses discos, inseridos no centro de placas de Petri, dessa vez, em meio de cultura sólido, com extrato de malte (2\%)/ágar $(1,5 \%)$ e diferentes concentrações de ametrina $\left(0,15,65,142 \mathrm{mg} . \mathrm{kg}^{-1}\right)$, possuindo como barreira Ciênc. agrotec., Lavras. V.27, n.4, p.840-845, jul./ago., 2003 
física papel celofane. $\mathrm{O}$ experimento foi conduzido em triplicata, sendo o fungo incubado por sete dias, em estufa à temperatura de $25^{\circ} \mathrm{C}$.

\section{Avaliação do Crescimento Fúngico}

Os extratos fúngicos foram obtidos congelandose a massa micelial com nitrogênio líquido e liofilizando. Ao produto liofilizado obtido, foi adicionado etanol $\left(95^{\circ} \mathrm{GL}\right)$, na proporção de $0,03 \mathrm{~g}$ de massa liofiliza$\mathrm{da} / \mathrm{mL}$ de etanol, aquecendo-se a $50^{\circ} \mathrm{C}$, durante $5 \mathrm{minu}$ tos. A solução obtida foi submetida à filtração simples. $\mathrm{O}$ procedimento foi repetido por mais duas vezes. O extrato fúngico obtido foi evaporado à secura em evaporador rotativo, à temperatura de $40^{\circ} \mathrm{C}$, e ao final, foram adicionados $6 \mathrm{~mL}$ de etanol para a recuperação do extrato e acondicionado em frascos protegidos de luz e sob refrigeração a $-5^{\circ} \mathrm{C}$, para a quantificação do teor de ergosterol.

$\mathrm{O}$ crescimento do micélio fúngico foi avaliado pela medida do teor de ergosterol.

Para a obtenção do ergosterol, foi utilizada a metodologia modificada descrita por Anderson et al. (1994). O extrato fúngico obtido na extração alcoólica foi saponificado com $\mathrm{KOH}(60 \mathrm{~g} / 100 \mathrm{~mL})$, durante 30 minutos, à temperatura de $90^{\circ} \mathrm{C}$, na proporção de $0,04 \mathrm{~g}$ de massa liofilizada/ $\mathrm{mL}$ de solução de $\mathrm{KOH}$. Após saponificação, a água e o etanol residual foram retirados utilizando-se de evaporador rotativo, a uma temperatura de $40^{\circ} \mathrm{C}$. Ao saponificado, foi adicionado hexano/água (4:1) para o fracionamento. Após a retirada da fase orgânica, foram adicionados mais $10 \mathrm{~mL}$ de hexano para um segundo fracionamento. A fração orgânica foi evaporada em evaporador rotativo e o ergosterol recuperado foi armazenado em metanol, na ausência de luz, a $-5^{\circ} \mathrm{C}$.

A quantificação do ergosterol foi realizada em aparelho de cromatografia líquida de alta eficiência (CLAE), marca Agilent, modelo HP 1100, utilizando uma coluna C18 (Zorbax C-18 $225 \mathrm{~mm}$ ), detetor de arranjo de diodos (DAD), em comprimento de onda 282 $\mathrm{nm}$, tendo como fase móvel metanol a um fluxo de 1 mL. min $^{-1}$. Para as análises, uma curva analítica (ergosterol - Sigma Chemical Co.) foi preparada.

\section{Análise Estatística}

O delineamento estatístico foi inteiramente casualizado, empregando fatorial $3 \times 4$, ou seja, 3 tempos de incubação (2, 5 e 7 dias), concentrações do herbicida ametrina $\left(0,15,65\right.$ e $\left.142 \mathrm{mg} . \mathrm{kg}^{-1}\right)$ com 3 repetições. Os valores obtidos foram trabalhados pelo Sistema de Análise Estatística - SANEST (Zonta \& Machado, 1991).

\section{RESULTADOS E DISCUSSÃO}

$\mathrm{O}$ meio extrato malte proporcionou bom crescimento e produção de pigmento, parâmetros importantes dentro do presente trabalho.

O papel celofane, como barreira física, não impediu que o fungo buscasse seus nutrientes no meio de cultura, e se desenvolveu, em sua maioria, na superfície do papel celofane, propiciando um bom crescimento do fungo e uma recuperação eficaz do mícelio.

A análise de variância indica resposta significativa, pelo teste $\mathrm{F}$, para os dois tratamentos (tempo e concentração de herbicidas) e interação tempo x concentração de herbicida para o teor de ergosterol.

A resposta significativa dos tratamentos mostra que a medida do crescimento fúngico, pela quantificação do teor de ergosterol, é um método eficaz para essa finalidade, conforme relatado na literatura (Seitz et al.,1977, 1979; Ruzicka et al., 2000).

A Figura 1 mostra o comportamento do crescimento micelial em relação ao teor de ergosterol, quando o $F$. oxysporum foi incubado aos cinco e sete dias, a várias concentrações de herbicida.

Observa-se uma redução do teor de ergosterol, aos 5 dias de incubação, à medida que se aumenta a concentração do herbicida. Todavia, aos 7 dias de incubação, houve um aumento do teor de ergosterol, para a concentração de herbicida de $142 \mathrm{mg} \cdot \mathrm{kg}^{-1}$, o que pressupõe uma alteração no metabolismo do fungo, ou ainda, uma alteração nas propriedades físico-químicas do herbicida, tais como a cristalização (solubilidade é de $200 \mathrm{mg} \cdot \mathrm{kg}^{-1}$ ) ou a formação de micélias.

Os resultados do teor de ergosterol, aos dois dias de incubação não se ajustaram à análise de regressão e mostraram comportamento diferente dos outros dias de incubação. Esses resultados são apresentados na Tabela 1.

TABELA 1 - Valores médios do teor de ergosterol, aos 2 dias de incubação.

\begin{tabular}{ccccc}
\hline \multirow{2}{*}{ Parâmetros } & \multicolumn{4}{c}{ Concentração de herbicida } \\
\cline { 2 - 5 } & $\mathbf{0}$ & $\mathbf{1 5}$ & $\mathbf{6 5}$ & $\mathbf{1 4 2}$ \\
\hline Ergosterol & 10,667 & 1,0910 & 0,8660 & 0,8603 \\
\hline
\end{tabular}




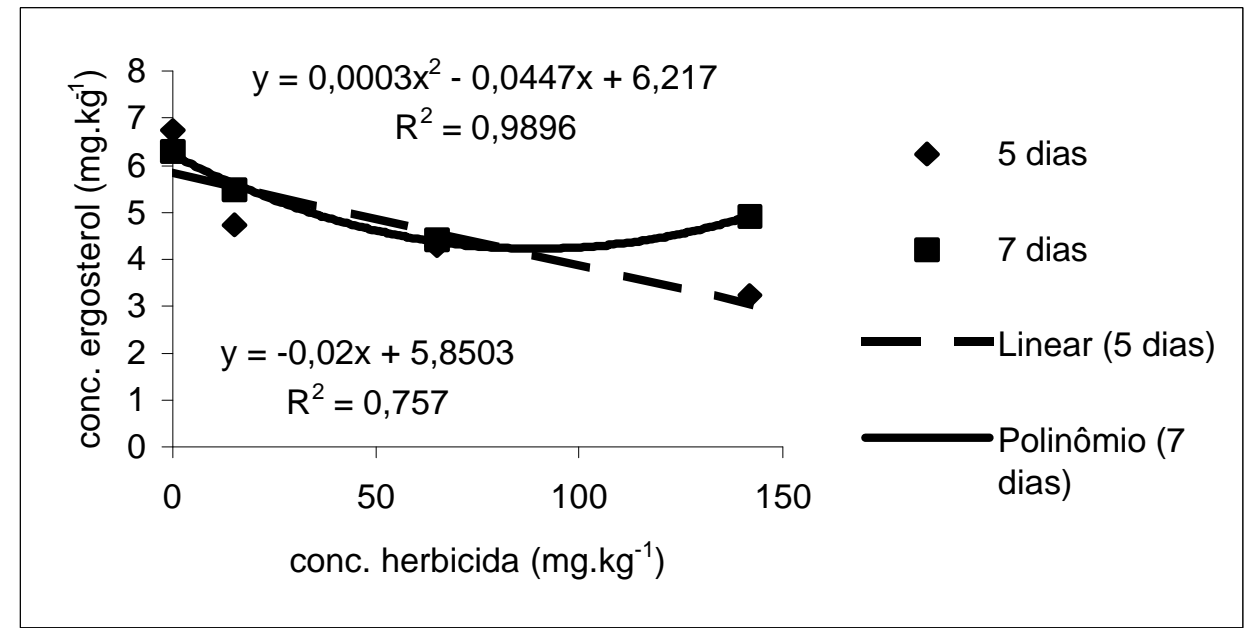

FIGURA 1 - Curva e equações de regressão representativas do teor de ergosterol obtido do crescimento micelial de Fusarium oxysporum, em função de diferentes concentrações de herbicidas.

Em relação ao teor de ergosterol, em 2 dias de incubação, praticamente não houve aumento no crescimento micelial até a concentração de herbicida de 15 mg.kg-1 e uma redução no crescimento de $18,82 \%$ (em relação à concentração zero), quando na concentração de herbicida de $65 \mathrm{mg} . \mathrm{kg}^{-1}$, mantendo-se praticamente constante na concentração de $142 \mathrm{mg} \cdot \mathrm{kg}^{-1}$.

Portanto, pode-se constatar que para todos os dias de incubação (2, 5 e 7 dias) a concentração de herbicida que provocou a maior redução no crescimento micelial foi a de $65 \mathrm{mg} \cdot \mathrm{kg}^{-1}$. O melhor tempo para se medir o crescimento micelial é de cinco dias, uma vez que houve diminuição da concentração de ergosterol.

\section{CONCLUSÃO}

Pelos resultados obtidos experimentalmente, evidencia-se uma boa correlação entre o conteúdo de ergosterol e biomassa fúngica metabolicamente ativa. $\mathrm{O}$ teor de ergosterol pode indicar o crescimento fúngico e, dessa forma, ser utilizado como bioindicador de contaminação por herbicida.

$\mathrm{O}$ crescimento fúngico foi inibido, segundo o parâmetro medido, com aumento da concentração do herbicida ametrina. Os dados determinam equações de regressão lineares, no quinto dia de incubação, para essa forma de medida de crescimento do fungo, na concentração de $65 \mathrm{mg} \cdot \mathrm{kg}^{-1}$. Pode-se dessa forma, inferir que o tempo de cinco dias é suficiente para as análises da res- posta fúngica, quando seu crescimento ocorre em meio controlado, contaminado pelo herbicida.

\section{REFERÊNCIAS BIBLIOGRÁFICAS}

ANDERSON, P.; DAVIDSON, C. M.; LITTLEJOHN, D.; URE, A. M.; SHAND, C. A.; CHESHIRE, M. V. Extraction of ergosterol fron peaty soils and determination by high performance liquid chromatography. Talanta, Amsterdam, v. 41, n. 5, p. 711-720, May 1994.

CHRISTENSEN M. A view of fungal ecology. Mycologia, New York, v. 81, n. 1, p. 1-19, Jan./Feb. 1989.

COURA, S. M. C.; GUERREIRO, M. C.; SOUZA, J. A.; LIMA, J. M.; SIQUEIRA, J. O.; SOUZA, P. E.; CARDOSO, M. G.; ELISA, E. Fusarium oxysporum como bioindicador de herbicida a base de ametrina. In: ENCONTRO REGIONAL DA SOCIEDADE BRASILEIRA DE QUÍMICA, 14., 2000. Anais... [S.1.: S.n.], 2000.

DAVIS, M. W.; LAMAR, R. T. Evaluation of methods to extract ergosterol for soil fungal biomass. Soil Biology and Biochemistry, Oxford, v. 24, n. 3, p. 189-198, Mar. 1992.

DEACON, J. W. Structure and ultrastructure: modern mycology. Cambridge: [s.n.], 1998. 35 p. 
ELLIS, M. B.; ELLIS, J. P. Plurivorous fungi on herbaceous plants: microfungi on land plants: an identification handbook. England: [s.n.], 1997. 293 p.

FRANKLAND, J. C. Ecological methods of observing and quantifying soil fungi. Transactions of the Mycological Society of Japan, Tokyo, v. 31, p. 89-101, 1990.

FRANKLAND, J. C.; DIGHTON, J.; BODDY, L. Methods for studying fungi in soil and forest litter. Methods in Microbiology, Oxford, v. 22, p. 343-404, 1990.

GOULSTON, G.; MERCER, E. I. ERGOSTA $5,7,24(28)$ - Trien - 3 $\beta$ - ol, a new intermediate in ergosterol biosiynthesis in Phycomyces blakeslleanus. Phytochemistry, Oxford, v. 8, n. 10, p. 1945-1948, Oct. 1969.

GRANT, L. F.; HARVEY, A. E. Quantitative measuarement of ectomycorrhizae on plant roots. In: SCHENK, N. C. (Ed.). Methods and principles of mycorrhizal reasearch. Saint Paul: The American Phytopathological Society, 1982. cap. 14, p. 157-164.

HASELWANDTER, K.; BERRECK, M. Fungi as bioindicators of radiocesium contamination: pre and post chernobyl activities. Transctions of the Bristish Mycological Society, Cambridge, v. 90, p. 171-174, 1988.

HÖLKER, U.; FAKOUSSA, R. M.; HÖFER, M. Growth substrates control the ability of Fusarium oxysporum to solubilize low-rank coal. Applied Microbiology and Biotechnology, New York, v. 44, n.3/4, p. 351-355, Dec. 1995.

MARTIN, F.; DELARUELLE, C.; HILBERT, J. L. An improved ergosterol assay to estimate fungal biomass in ectomycorrhizas. Mycological Research, Cambridge, v. 94, n. 9, p. 1059-1064, Sept. 1990.

MEDENTSEV, A. G.; AKIMENKO, V. K. Naphtoquinone Metabolites of the fungi. Phytochemistry, Oxford, v. 47, n. 6, p. 935-959, Mar. 1998.

NELSON, P. E.; TOUSSOUN, T. A.; MARASAS, W. F. O. Fusarium species: a illustrated manual for identi- fication. Pennsylvania: Pennsylvania State University, 1983. $193 \mathrm{p}$.

NEWEL, S. Y. Estimating fungal biomass and productivy in decomposing litter. In: The fungal community: its organization and role in the ecosystem. New York: Dekker, 1992. p. 521-561.

PAOLETTI, M. G. Using bioindicators based on biodiversity to assess landscape sustainability. Agriculture, Ecosystems \& Environment, Amsterdam, v. 74, n. 1/3, p. 1-18, June 1999.

PARKINSON, D. Filamentous fungi. In: Methods of soil analysis: soil science society of America. Madison: SSSA, 1982. part 2, p. 949-968.

PEINTNER, U.; MOSER, M. Survey of heavy metal deposition at the schulterberg (Achenkirch Altitude Profile) by using basidiomycetes as bioindicators. Phyton - Annales Rei Botanicae, Horn, v. 35, n. 4, p. 155$162,1996$.

RICHARDSON, D. H. S. Biological indicators of pollution. Dublin: Royal Irish Academy, 1987. 242 p.

RUZICKA， S.; EDGERTON， D.; NORMAN， M.; HILL, T. The Utility of ergosterol as a bioindicator of fungi in temperate soils. Soil Biology \& Biochemistry, Oxford, v. 32, n. 7, p. 989-1005, July 2000.

SEITZ, L. M.; MOHR, H. E.; BURROUGHS, R.; SAUER, D. B. Ergosterol as an indicator of fungal invasion in grains. Ceral Chemistry, Saint Paul, v. 54, n. 6, p. 1207-1217, 1977.

SEITZ, L. M.; MOHR, H. E.; BURROUGHS, R.; SAUER, D. B.; HUBBARD, J. D. Ergosterol as a Measure of fungal growth. Phytopathology, Saint Paul, v. 69, n. 11, p. 1202-1203, Nov. 1979.

SODERSTROM, B. E. Vital staining of fungi in pure culture and in soil with fluorescein diacetate. Soil Biology and Biochemestry, Oxford, v. 9, n. 1, p. 59-63, 1977.

ZONTA, E. T.; MACHADO, A. A. Manual de estatística para microcomputadores. Pelotas: UFPel, 1991. $102 \mathrm{p}$. 